\title{
Comparison of the efficacy and safety of hybrid and sequential therapies as a first-line regimen for Helicobacter pylori infection in Turkey
}

Ayşe Kefeli ${ }^{1}$, Sebahat Başyigit² ${ }^{2}$ Abdullah Ozgur Yeniova ${ }^{3}$, Serdar Ozkan ${ }^{4}$, Yasar Nazligul ${ }^{2}$

\begin{abstract}
${ }^{1}$ Gastroenterology Department, Siirt State Hospital, Siirt, Turkey
${ }^{2}$ Gastroenterology Department, Kecioren Training Hospital, Ankara, Turkey

${ }^{3}$ Gastroenterology Department, Gaziosmanpasa University, Tokat, Turkey

${ }^{4}$ Thoracic Surgery, Siirt State Hospital, Siirt, Turkey
\end{abstract}

Submitted: 12 October 2015

Accepted: 7 December 2015

Arch Med Sci 2018; 14, 2: 276-280

DOI: 10.5114/aoms.2016.58595

Copyright $\odot 2016$ Termedia \& Banach

\author{
Corresponding author: \\ Ayşe Kefeli PhD \\ Gastroenterology Department \\ Siirt State Hospital \\ Siirt Devlet Hastanesi, \\ Merkez \\ 56100 Siirt, Turkey \\ Phone: +90 5054506997 \\ E-mail: \\ aysekefeli@hotmail.com
}

\section{Abstract}

Introduction: Helicobacter pylori infection is a common infection worldwide. The most frequently recommended treatment for eradication of $H$. pylori remains triple therapy. In this study, we compared sequential and hybrid regimens for $H$. pylori eradication in a region of Turkey with high resistance to clarithromycin.

Material and methods: Three hundred and forty $H$. pylori-positive patients were enrolled in the study. The subjects were randomly divided into two groups. The first group (170 patients) received rabeprazole $(40 \mathrm{mg} / \mathrm{b}$.i.d.) and amoxicillin (1000 mg/b.i.d.) for 2 weeks and metronidazole and clarithromycin $(500 \mathrm{mg} / \mathrm{b} . \mathrm{i} . \mathrm{d}$.) during the second week in the hybrid therapy group. The second group (170 patients) received rabeprazole $(40 \mathrm{mg} / \mathrm{b}$.i.d.) for 14 days, amoxicillin (1000 mg/b.i.d.) for the first 7 days, and metronidazole plus clarithromycin (each $500 \mathrm{mg} /$ b.i.d.) during the next 7 days in the sequential therapy group.

Results: In the per-protocol analysis, the eradication rate in the hybrid therapy group was $96.1 \%(147 / 153)$, and in the sequential therapy group it was $90.9 \%(140 / 154)$. There was no significant difference between the two groups $(p=0.06)$. Ninety-seven of those 340 patients reported minor adverse drug reactions. The percentages of patients with adverse reactions were $30.6 \%$ in the hybrid therapy group and $26.5 \%$ in the sequential therapy group $(p=0.74)$.

Conclusions: Both therapies are highly effective for eradication of $\mathrm{H}$. pylori, and could be recommended as a first-line therapy in regions with high antibiotic resistance.

Key words: Helicobacter pylori, hybrid regimen, sequential regimen.

\section{Introduction}

Helicobacter pylori infection is a common infection worldwide and is well recognized to be the main cause of gastritis, peptic ulcers, and gastric cancer [1]. Triple therapy, which contains a proton pump inhibitor (PPI) and amoxicillin with either clarithromycin or metronidazole, is a standard first-line eradication regimen $[2,3]$. However, in most countries, the eradication success of triple therapy has been reported to have decreased below acceptable levels $(\leq 80 \%)$ because of the increasing 
rate of $H$. pylori resistance to antibiotics $[4,5]$. Therefore, several regimens have been proposed as alternative first-line treatments for $H$. pylori infection, including sequential therapy (ST) and hybrid therapy $(\mathrm{HT})$.

Sequential therapy consists of a PPI (b.i.d) and amoxicillin $1 \mathrm{~g}$ (b.i.d) given for 7 days, followed by the PPI (b.i.d), clarithromycin $500 \mathrm{mg}$ (b.i.d), and metronidazole $500 \mathrm{mg}$ (b.i.d) for 7 days. Hybrid therapy is similar with the exception that the amoxicillin $1 \mathrm{~g}$ (b.i.d) is continued throughout the 14 days. According to recent studies, these therapies are highly effective and safe, but there are not enough data about the efficacy of these treatments in countries with high clarithromycin and metronidazole resistance rates [6-17].

However, ST and HT regimens both have been reported to be effective. They have little difference in treatment modality. It has been a subject of research whether there is a difference in the eradication rates between the two regimens. In the literature, there have been limited data comparing the differences in the efficacy and safety between these protocols, and conflicting results have been reported.

In this study, we aimed to assess and compare the efficacy and safety of ST and HT protocols in H. pylori eradication in Turkey in a region that has high clarithromycin and metronidazole resistance rates.

\section{Material and methods}

\section{Subjects}

This prospective, single-center study was performed at the Gastroenterology Department of Siirt State Hospital in Turkey, from October 2014 to April 2015. Patients who had biopsy-proven H. pylori gastritis were eligible for the study. Exclusion criteria included previous attempts at $H$. pylori eradication therapy, recent use of antibiotic or bismuth salts or proton-pump inhibitors in the last 2 months before the study, chronic use of nonsteroidal anti-inflammatory drugs or corticosteroids, severe comorbid diseases, gastric malignancy including adenocarcinoma and lymphoma, pregnancy or lactation, diarrhea, prior gastric surgery, allergy to any of the drugs in the current treatment, and age under 18 years. Informed consent was obtained from each patient before enrolling them in the study.

The patients were randomly given ST and HT protocols. Patients in the ST group were given rabeprazole $40 \mathrm{mg}$ (b.i.d, $30 \mathrm{~min}$ before meals) and amoxicillin $1000 \mathrm{mg}$ (b.i.d, $1 \mathrm{~h}$ after meals) for the first 7 days of the treatment period and then rabeprazole $40 \mathrm{mg}$ (b.i.d, $30 \mathrm{~min}$ before meals), clarithromycin $500 \mathrm{mg}$ (b.i.d, $1 \mathrm{~h}$ after meals), and metronidazole $500 \mathrm{mg}$ (b.i.d, $1 \mathrm{~h}$ after meals). During the remaining 7 days, patients in the HT group were given the same treatment with the exception that amoxicillin $1 \mathrm{~g}$ (b.i.d) was continued up to the $14^{\text {th }}$ day.

A detailed written treatment protocol was given to all patients to prevent misuse of medications. They were actively interviewed about side effects and treatment adherence using a structured questionnaire 1 week after the end of the treatment. To confirm patient compliance, we asked the patients to bring their remaining medication and counted the rest of their pills. Patients with a compliance rate of less than $80 \%$ were excluded from the study per-protocol (PP) analysis. Eradication rates of $H$. pylori were measured by the ${ }^{13} \mathrm{C}$-urea breath test $\left({ }^{13} \mathrm{C}\right.$-UBT). Six weeks after the treatment period, and after at least 2 weeks with no administration of PPI, we confirmed $H$. pylori eradication using ${ }^{13} \mathrm{C}-\mathrm{UBT}$.

\section{Statistical analysis}

Both PP (excluding patients with poor compliance of therapy and patients with unavailable data after therapy) and intention to treat (ITT) (including all eligible patients enrolled in the study regardless of compliance with the study protocol; patients with unavailable data are assumed to have been unsuccessfully treated) analyses were used to evaluate the $H$. pylori eradication rate. Data analysis was performed using the statistical software package program IBM SPSS for Windows (version 19.1; SPSS Inc., Chicago, IL, USA). Intergroup comparisons of categorical variables were done using the $\chi^{2}$ test, and continuous variables were compared using Student's t-test. Categorical variables were presented as percentages or counts, and continuous variables were presented as means and standard deviations in the descriptive analysis. By setting the significance level to $p$ $<0.05$, the statistical power to $90 \%$, and the dropout rate to $10 \%$, we calculated a need for 170 patients in each group.

\section{Results}

\section{Patient characteristics}

There was no significant difference between the average ages of the groups, which were 37.2 \pm 13.2 in the ST group and $38.1 \pm 12.1$ in the HT group ( $p=0.50)$. The gender distribution was also similar between groups $(p=0.51)$. There was no significant difference in relation to endoscopic findings between the groups $(p=0.69)$ (Table I).

\section{Eradication rate for first-line treatment}

In the ITT analysis, the eradication rates were $82.4 \%(140 / 170)$ for the ST group and $86.5 \%$ (147/170) for the HT group. There was no signifi- 
Table I. Characteristics of patients

\begin{tabular}{|lccc|}
\hline Parameter & HT & ST & P-value \\
\hline Age [year] & $38.08 \pm 12.0$ & $37.19 \pm 13.2$ & 0.5 \\
\hline \begin{tabular}{l} 
Gender: \\
\hline Male
\end{tabular} & $50.6 \%$ & $54.1 \%$ & 0.51 \\
\hline Female & $49.4 \%$ & $45.9 \%$ & \\
\hline \begin{tabular}{l} 
Diagnosis: \\
\hline Gastric ulcer
\end{tabular} & $14.1 \%$ & $11.2 \%$ & \\
\hline \begin{tabular}{l} 
Duodenal ulcer \\
\hline Gastritis
\end{tabular} & $19.4 \%$ & $21.2 \%$ & \\
\hline \begin{tabular}{l} 
Consumption: \\
\hline Smoking
\end{tabular} & $196.5 \%$ & $67.6 \%$ & \\
\hline Alcohol & $2.4 \%$ & $2.9 \%$ & 0.73 \\
\hline
\end{tabular}

HT - hybrid therapy, ST - sequential therapy.

cant difference between the two groups $(p=0.29)$ (Table II).

The PP analysis was performed on 154 patients in the ST group and 153 patients in the HT group. We excluded patients who did not come for UBT (10 patients in the ST group and 9 patients in HT group) and who had poor adherence to the treatment ( $n=6$ in the ST group and 8 in the HT group). In the PP analysis, the eradication rates were $90.9 \%(140 / 154)$ for the ST group and $96.1 \%$ (147/153) for the HT group. There was no significant difference in eradication rates between the two groups ( $p=0.06)$ (Table III, Figure 1$)$.

\section{Adverse drug reactions}

Ninety-seven of the 340 patients reported minor adverse drug reactions. The percentages of patients with adverse reactions were $26.5 \%$ $(45 / 170)$ in the ST group and $30.6 \%(52 / 170)$ in the HT group $(p=0.74)$. The percentages of patients who discontinued the treatment due to ad-
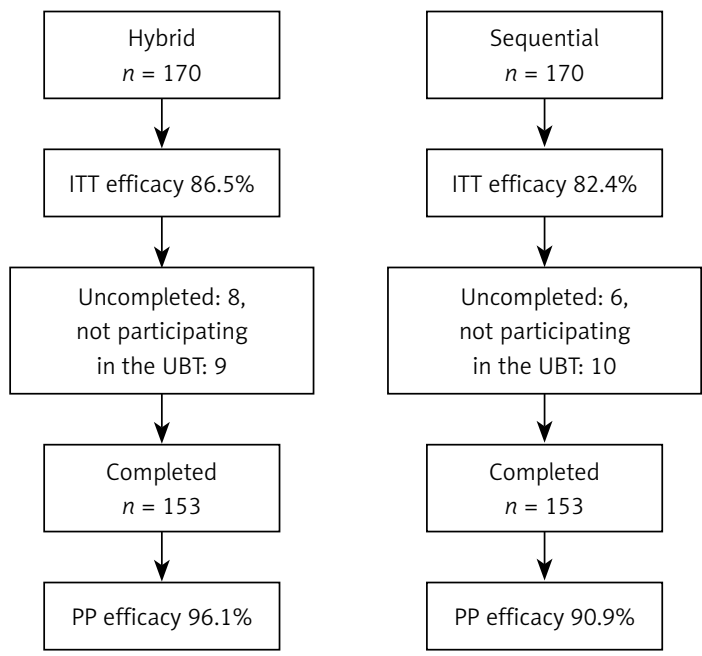

Figure 1. Hybrid and sequential therapies ITT - intention to treat, PP - per protocol.
Table II. Helicobacter pylori eradication rates in the 14-day hybrid and sequential therapies

\begin{tabular}{|lccc|}
\hline Variable & $\begin{array}{c}\text { HT group, } \\
\text { rate } \%(n)\end{array}$ & $\begin{array}{c}\text { ST group, } \\
\text { rate } \%(n)\end{array}$ & $P$-value \\
\hline ITT analysis & $\begin{array}{c}86.5 \% \\
(147 / 170)\end{array}$ & $\begin{array}{c}82.4 \% \\
(140 / 170)\end{array}$ & 0.29 \\
\hline PP analysis & $\begin{array}{c}96.1 \% \\
(147 / 153)\end{array}$ & $\begin{array}{c}90.9 \% \\
(140 / 154)\end{array}$ & 0.06 \\
\hline
\end{tabular}

HT - hybrid therapy, ST - sequential therapy, ITT - intention to treat, PP - per protocol.

Table III. Adverse events in the hybrid and sequential therapies

\begin{tabular}{|lccc|}
\hline Parameter & HT & ST & $P$-value \\
\hline Pruritus & $16(9.4 \%)$ & $12(7.1 \%)$ & 0.28 \\
\hline Bitter taste & $14(8.2 \%)$ & $10(5.9 \%)$ & 0.31 \\
\hline Loose stool & $12(7.1 \%)$ & $15(8.8 \%)$ & 0.43 \\
\hline Headache & $7(4.1 \%)$ & $4(2.4 \%)$ & 0.38 \\
\hline $\begin{array}{l}\text { Abdominal } \\
\text { pain }\end{array}$ & $3(1.8 \%)$ & $4(2.4 \%)$ & 0.57 \\
\hline Total & $53(30.6 \%)$ & $45(26.5 \%)$ & 0.74 \\
\hline
\end{tabular}

HT - hybrid therapy, ST - sequential therapy.

verse events were $3.5 \%(6 / 170)$ in the ST group and $4.7 \%(8 / 170)$ in the HT group.

In order of frequency there were bitter taste, loose stools, and pruritus as the most common adverse reactions in all study groups. Pruritus was the most common adverse reaction in the HT group, and loose stools was the most common event in the ST group. However, these differences were not statistically significant, and there were no major life-threatening adverse reactions (Table III).

\section{Discussion}

In the present study, we assessed and compared the efficacy and safety of the ST and the HT protocols as a first-line treatment of $H$. pylori infection in Turkey, which has high clarithromycin and metronidazole resistance rates. We found satisfactory results, which indicate that the eradication rates were $90.9 \%$ and $82.4 \%$ for the ST group and $96.1 \%$ and $86.5 \%$ for the HT group in the PP and ITT analyses, respectively. There was no significant difference in eradication rates between the two groups.

The European $H$. pylori study has suggested that treatment regimens should achieve an eradication rate over $80 \%$ on ITT analysis and $85 \%$ on PP analysis in order to be acceptable as first-line therapy for $H$. pylori eradication [18]. The efficacy of H. pylori eradication regimens was also classified based on per-protocol success as follows: A, excellent (> 95\%); B, good (90-95\%); C, fair (85-89\%); D, bad (81-84\%); and E, unacceptable (<80\%) [19]. According to this scale, our results showed excellent efficacy (grade A) for the HT protocol and good efficacy (grade B) for the ST protocol. 
Since the efficacy of standard triple therapy decreased to an unacceptable level for successful eradication rates in most countries, new strategies have been developed in the last decade [5]. The main factor leading to the high eradication failure rate is increased antibiotic resistance of the bacteria [20]. The prevalence of antibiotic resistance varies considerably by region and is related to the use of antibiotics. Especially, recent data suggest that clarithromycin resistance is a growing problem affecting the eradication rate of $H$. pylori. However, studies from different countries that have high clarithromycin resistance rates have reported that eradication rates with $\mathrm{HT}$ ranged from $76.4 \%$ to $97.4 \%[8,13]$. It has been shown in a meta-analysis that the cure rate of the infection is reduced to $55 \%$ in patients with clarithromycin resistance [21].

The rate of resistance to clarithromycin has increased gradually in Turkey. Recently, a study on a Turkish dyspeptic population revealed a clarithromycin resistance rate of $40.2 \%$, using the PCR method [22]. Metronidazole resistance is the other main factor responsible for eradication failure in Turkey; a recent study reported a metronidazole resistance rate of $45.5 \%$ [23]. This increased incidence of high-level clarithromycin and metronidazole resistance in $H$. pylori is a serious concern, as it negatively affects eradication regimens.

To overcome increasing resistance and eradication failures, many alternative therapeutic strategies that utilize adjusted drug combination, dosage, duration, and timing of the drug administration have been studied. Among these therapies, ST and HT protocols have emerged as the most promising treatments and have been reported to be effective [6-12]. In these protocols, amoxicillin is administered before other antibiotics. Amoxicillin disrupts the bacterial cell wall of $H$. pylori and enables clarithromycin and metronidazole to infiltrate into the bacteria. Due to the disrupted cell wall, $H$. pylori is unable to form an efflux pump to export clarithromycin, and the effect of the antibiotics can be maintained and is effective, even in cases of clarithromycin-resistant $H$. pylori [24-27].

Eradication rates also appear to have significant regional variation. In Turkey, some previous studies were conducted that compared triple therapy and ST; they showed various PP eradication rates ranging from $57 \%$ to $88 \%$ in ST, which was more effective than triple therapy [22, 28-30]. In addition, ST has been reported to be more effective than triple therapy by studies from Asian countries [31, 32] and many other countries with high clarithromycin resistance rates by meta-analysis [33].

Both ST and HT protocols contain the same drugs, but amoxicillin is used 7 more days in the HT protocol. Because of this difference, studies have been designed around the differences in the effectiveness of the ST and the HT regimens. How- ever, they have reported conflicting results. Some of them from Taiwan [13], which also has high antibiotic resistance rates, and from Korea have shown no significant differences between the two protocols. One of the Italian studies [7] also showed no significant differences between them, while the other [9] reported that the eradication rate of the HT group was lower than that of the ST group. A recent study from Iran [8] reported that the eradication rate of the HT group was higher than that of the ST group. In the present study, we found that the eradication rate of the HT group was slightly higher than that of the ST group, but this difference was not statistically significant. Our results concur with those of the majority of the previous results.

An acceptable eradication regimen needs good adherence to the treatment and a low incidence of serious adverse events (less than 5\%) [34]. Although both regimens require a complex schedule in which the administered drugs should be changed in the second week, and even though there is a concern that these protocols could cause possible misuse, in our study we observed good compliance in both groups. It may also be thought that these protocols can lead to high incidence of adverse events because they contain multiple drugs. In our study, only 14 (4.1\%) patients in all study groups discontinued the therapy because of adverse reactions. However, $28.5 \%$ of all patients who experienced minor adverse events completed the treatment. Despite the longer administration duration of amoxicillin in the HT protocol, the compliance rates did not differ significantly between the two groups $(4.7 \%$ in the HT group and $3.5 \%$ in the ST group).

The major limitation of our study was the lack of assessment of primary resistance to different antibiotics for $H$. pylori strains. However, our main purpose was to assess the two novel regimens as first-line therapy in clinical practice, and it is not recommended to assay antibiotic resistance tests in first-line treatment.

In conclusion, the HT and ST protocols provide acceptable eradication rates in a region with high resistance to clarithromycin and metronidazole. Both therapies could be recommended as a firstline therapy with high efficacy and safety, and hybrid therapy, which is a novel therapy, could be an alternative to sequential therapy, but further studies are needed to substantiate these findings.

\section{Conflict of interest}

The authors declare no conflict of interest.

\section{References}

1. McColl KE. Clinical practice Helicobacter pylori infection. N Engl J Med 2010; 362: 1597-604. 
2. Malfertheiner P, Megraud F, O'Morain CA, et al. Management of Helicobacter pylori infection: the Maastricht IV/ Florence Consensus Consensus Report. Gut 2012; 61: 646-64.

3. Fock KM, Katelaris P, Sugano K, et al. Second Asia-Pacific Consensus Guidelines for Helicobacter pylori infection. J Gastroenterol Hepatol 2009; 24: 1587-600.

4. Graham DY, Fischbach L. Helicobacter pylori treatment in the era of increasing antibiotic resistance. Gut 2010; 59: 1143-53.

5. Gisbert JP, Calvet X. Review article: the effectiveness of standard triple therapy for Helicobacter pylori has not changed over the last decade, but it is not good enough. Aliment Pharmacol Ther 2011; 34: 1255-68.

6. Hsu PI, Wu DC, Wu JY, Graham DY. Modified sequential Helicobacter pylori therapy: proton pump inhibitor and amoxicillin for 14 days with clarithromycin and metronidazole added as a quadruple (hybrid) therapy for the final 7 days. Helicobacter 2011; 16: 139-45.

7. De Francesco V, Hassan C, Ridola L, Giorgio F, lerardi E, Zullo A. Sequential, concomitant and hybrid first-line therapies for Helicobacter pylori eradication: a prospective randomized study. J Med Microbiol 2014; 63: 748-52.

8. Sardarian H, Fakheri H, Hosseini V, Taghvaei T, Maleki I, Mokhtare M. Comparison of hybrid and sequential therapies for Helicobacter pylori eradication in Iran: a prospective randomized trial. Helicobacter 2013; 18: 129-34.

9. Zullo A, Scaccianoce G, De Francesco V, et al. Concomitant, sequential, and hybrid therapy for $\mathrm{H}$. pylori eradication: a pilot study. Clin Res Hepatol Gastroenterol 2013; 37: 647-50.

10. Molina-Infante J, Romano M, Federico A, et al. 14-day, high-dose acid suppression, non-bismuth quadruple therapies (hybrid vs. concomitant) for Helicobacter pylori infection: a randomized trial. Gastroenterology 2013; 144: 242 (Abstract: Presented in Digestive Disease Week (DDW) 18-21 May, 2013 Orlando, FL).

11. Wu JY, Hsu PI, Wu DC, Graham D. Optimal duration to maintain greater than $95 \%$ eradication rate in hybrid therapy for H. pylori. Gastroenterology 2012; 142: 484 (Abstract: Presented in Digestive Disease Week (DDW) 19-21 May, 2012, San Diego).

12. Graham DY, Gisbert JP. Helicobacter pylori: tailored therapy with novel sequential quadruple therapies. Nat Rev Gastroenterol Hepatol 2013; 10: 6-8.

13. Oh DH, Lee $\mathrm{DH}$, Kang KK, et al. Efficacy of hybrid and sequential therapies as first line treatment for $\mathrm{H}$. pylori infection in Korea. J Gastroenterol Hepatol 2013; 28: 327 (Abstract: Presented in Asian Pacific Digestive Week (DDW) 2013 World Congress of Gastroenterology, 21-24 September 2013, Shanghai Expo Center, Shanghai, China).

14. Hsu P, Wu D, Wu J, Graham D. Search for a Grade A therapy for $\mathrm{H}$. pylori eradication: 14-day sequential or sequential concomitant hybrid therapy. Helicobacter 2010; 15: 314 (Abstract; Presented in XXIII International Workshop on Helicobacter and Related Bacteria in Chronic Digestive Inflammation and Gastric Cancer, 16-18 September, 2010, Rotterdam).

15. Molina-Infante J, Romano M, Fernandez-Bermejo $M$, et al. Optimized nonbismuth quadruple therapies cure most patients with Helicobacter pylori infection in populations with high rates of antibiotic resistance. Gastroenterology 2013; 145: 121-8.

16. He L, Deng T, Luo H. Meta-analysis of sequential, concomitant and hybrid therapy for Helicobacter pylori eradication. Intern Med 2015; 54: 703-10.

17. Wang B, Wang YH, Lv ZF, et al. Efficacy and safety of hybrid therapy for Helicobacter pylori infection: a sys- tematic review and meta-analysis. Helicobacter 2015; 20: 79-88.

18. Graham DY, Lu H, Yamaoka Y. A report card to grade Helicobacter pylori therapy. Helicobacter 2007; 12: 275-8.

19. Graham DY, Lee YC, Wu MS. Rational Helicobacter pylori therapy: evidence-based medicine rather than medicine-based evidence. Clin Gastroenterol Hepatol 2014; 12: $177-86$.

20. Romano M, Cuomo A, Gravina AG, et al. Empirical levofloxacin- containing versus clarithromycin-containing sequential therapy for Helicobacter pylori eradication: a randomised trial. Gut 2010; 59: 1465-70.

21. Dore MP, Leandro G, Realdi G, Sepulveda AR, Graham DY. Effect of pretreatment antibiotic resistance to metronidazole and clarithromycin on outcome of Helicobacter pylori therapy: a meta-analytical approach. Dig Dis Sci 2000; 45: 68-76.

22. Sezgin O, Aslan G, Altintaş E, Tezcan S, Serin MS, Emekdaş G. Detection of point mutations on $23 \mathrm{~S}$ rRNA of Helicobacter pylori and resistance to clarithromycin with PCR-RFLP in gastric biopsy specimens in Mersin, Turkey. Turk J Gastroenterol 2008; 19: 163-7.

23. Cağdaş U, Otağ F, Tezcan S, Sezgin O, Aslan G, Emekdaş G. Detection of Helicobacter pylori and antimicrobial resistance in gastric biopsy specimens. Bul Mic 2012; 46: 398-409.

24. Murakami K, Fujioka T, Okimoto T, Sato R, Kodama M, Nasu M. Drug combinations with amoxycillin reduce selection of clarithromycin resistance during Helicobacter pylori eradication therapy. Int J Antimicrob Agents 2002; 19: 67-70.

25. Zullo A, Rinaldi V, Winn S, et al. A new highly effective short-term therapy schedule for Helicobacter pylori eradication. Aliment Pharmacol Ther 2000; 14: 715-8.

26. Webber MA, Piddock LJ. The importance of efflux pumps in bacterial antibiotic resistance. J Antimicrob Chemother 2003; 51: 9-11.

27. De Francesco V, Margiotta M, Zullo A, et al. Clarithromycin resistant genotypes and eradication of Helicobacter pylori. Ann Intern Med 2006; 144: 94-100.

28. Yakut M, Çinar K, Seven G, Bahar K, Özden A. Sequential therapy for Helicobacter pylori eradication. Turk J Gastroenterol 2010; 21: 206-11.

29. Demir M, Ataseven H. The effects of sequential treatment as a first-line therapy for Helicobacter pylori eradication. Turk J Med Sci 2011; 41: 427-33.

30. Uygun A, Kadayifci A, Yesilova Z, Safali M, Ilgan S, Karaeren N. Comparison of sequential and standard triple-drug regimen for Helicobacter pylori eradication: a 14-day, open-label, randomized, prospective, parallel-arm study in adult patients with nonulcer dyspepsia. Clin Ther 2008; 30: 528-34.

31. Sirimontaporn N, Thong-Ngam D, Tumwasorn S, Mahachai V. Ten-day sequential therapy of Helicobacter pylori infection in Thailand. Am J Gastroenterol 2010; 105: 1071-5.

32. Gao XZ, Qiao XL, Song WC, Wang XF, Liu F. Standard triple, bismuth pectin quadruple and sequential therapies for Helicobacter pylori eradication. World J Gastroenterol 2010; 16: 4357-62

33. Losurdo G, Leandro G, Principi M, et al. Sequential vs. prolonged 14-day triple therapy for Helicobacter pylori eradication: the meta-analysis may be influenced by 'geographical weighting'. Int J Clin Pract 2015; 69: 1112-20.

34. Kim N, Kim JJ, Choe YH, Kim HS, Kim JI, Chung IS. Diagnosis and treatment guidelines for Helicobacter pylori infection in Korea. Korean J Gastroenterol 2009; 54: 269-78. 